\title{
SALUD COMUNITARIA Y ACCIÓN SOCIAL EN EL CONTROL EPIDEMIOLOGÍCO DEL TRACOMA INFANTIL, ESPAÑA (1932-1939)"
}

\author{
Josep Bernabeu-Mestre
}

Universidad de Alicante

\section{María Eugenia Galiana-Sánchez}

\author{
Universidad de Alicante
}

\begin{abstract}
RESUMEN
En el contexto de la endemia tracomatosa que afectó a diversas regiones españolas hasta la década de 1960, se analizan los determinantes epidemiológicos del tracoma infantil y su abordaje desde el modelo de asistencia preventiva que encerraba el concepto de salud comunitaria que se perfiló en el período de entreguerras. La detección precoz de casos, unida a actividades preventivas, educativas, terapéuticas y de inspección, como las que llevaron a cabo las enfermeras visitadoras, contribuyeron al control de la enfermedad. Los resultados reafirman la validez de las estrategias de intervención horizontal de mejora de las condiciones higiénicas y de los factores medioambientales que explicaban la prevalencia del tracoma.
\end{abstract}

PALABRAS CLAVE: Tracoma infantil. Epidemiología. Salud comunitaria. Salud escolar. Enfermería de salud pública. España, siglo XX (1932-1939).

* Este trabajo fue presentado en una primera versión en la 8th European Social Science History Conference (13-16 April 2010, Ghent, Belgium) dentro de la Network: Family and Demography. Session: A4FAM05 Measles and Other Childhood Diseases, con el título de «Epidemiological factors, community health, social action and childhood in contemporary endemic trachoma in Spain, 1919-1964»; y se ha desarrollado en el marco de los proyectos de investigación: «La lucha contra la desnutrición en la España contemporánea y el contexto internacional (1874-1975)» (HAR2009-13504-C02-01. Ministerio de Ciencia e Innovación), «La sanidad española en el contexto internacional. Conferencias sanitarias, Sociedad de Naciones y Organismos Internacionales (1851-1975)». Proyecto Consolider del Ministerio de Educación y Ciencia [HUM 2006-06098], «Género, ideología y salud: la enfermería de salud pública en la sanidad española contemporánea (1923-1959)» (GRE10-12), y el Programa Prometeo. Generalitat Valenciana (Prometeo/2009/122). 


\title{
COMMUNITY HEALTH AND SOCIAL ACTION IN THE EPIDEMIOLOGIC CONTROL OF INFANTILE TRACHOMA, SPAIN (1932-1939)
}

\begin{abstract}
Within the context of the endemic trachoma that affected different regions of Spain until the 1960s, this paper analyses the epidemiological determinants of child trachoma and its treatment, based on a preventive care model which incorporated the concept of community health that took shape during the interwar period. Early detection of cases, together with preventive measures, education, therapy and inspections, such as those carried out by visiting nurses, all helped to control the disease. Our results reaffirm the validity of the horizontal intervention strategies used for improving the sanitary conditions and environmental factors responsible for this prevalence of trachoma.
\end{abstract}

KEY WORDS: Child trachoma. Epidemiology. School health. Public health nursing, Spain (19321939).

\section{INTRODUCCIÓN}

El tracoma, enfermedad ocular causada por la bacteria Chlamydia trachomatis, representa en la actualidad una de las tres grandes causas de ceguera «evitable» ${ }^{1}$. Durante las primeras décadas del siglo XX, era una enfermedad endémica en muchas regiones de Europa $^{2}$. España era una de las naciones más afectadas, mostrando una zona endémica de tracoma que se extendía por

1 La enfermedad es endémica en 56 países. Se calcula que existen más de 6 millones de personas ciegas, visualmente discapacitadas o con riesgo inmediato de ceguera a causa del tracoma. Las zonas más afectadas se caracterizan por su pobreza y por no tener cubiertas necesidades básicas como la vivienda, los servicios de salud, el agua y el saneamiento (KONYAMA, K. (2004-2005), History of trachoma control in Asia, Revue internationale du trachome et de pathologie oculaire tropicale et subtropicale et de santé publique, 81-82, pp. 107-168; Wright, H., TURner, A. Y TAYLOR, H. (2008), Trachoma, The Lancet, 371, pp. 1945-1954; TAYLOR, H. (2008), Trachoma, a blinding scourge from the bronze age to the twenty-first century, Melbourne, Haddinton Press). En 1997, la OMS puso en marcha el programa GET 2020 para la eliminación mundial del tracoma causante de ceguera, y más recientemente se ha desarrollado la estrategia conocida como SAFE (corrección quirúrgica, administración de antibióticos, higiene y mejoras ambientales) (MARIOTTI, S.P. Y PRÜSS, A. (2000), The SAFE strategy Preventing trachoma. A guide for environmental sanitation and improved hygiene, Geneva, World Health Organization; ORGANiSATION MONDIALE DE LA SANTE (2010), Agir pour réduire l'impact mondial des maladies tropicales négligées. Premier rapport de l'OMS sur les maladies tropicales négligées, Genève, Organisation Mondiale de la Santé).

2 Los datos recogidos en las comunicaciones y ponencias presentadas en el XIII Congreso Internacional de Oftalmología que tuvo lugar en Ámsterdam en 1929, subrayaban la incidencia que mostraba la enfermedad en los países de la Europa mediterránea (ARJONA TRAPOTE, J. Y ALONSO DE Medina y Bono, F. (1941), El tracoma, Madrid, Editorial Aldecoa, pp. 16-19). 
todo el litoral mediterráneo, desde la provincia de Gerona hasta Alicante, Murcia y Almería ${ }^{3}$.

En 1933, la Dirección General de Sanidad hacía públicos los datos de la campaña antritracomatosa de 1932 y aportaba unas cifras que superaban los 50.000 tracomatosos, aunque se sospechaba que podían alcanzar los 100.000, con unas tasas que oscilarían para el conjunto nacional entre el 2,12 y el 4,24 por mil habitantes. Las más afectadas eran las mujeres con un $52,6 \%$, y por grupos de edades, la infancia aparecía en primer lugar con un $39,7 \%$, seguida del grupo de adultos $(32,6 \%)$ y de los adolescentes con un $28 \%{ }^{4}$.

En investigaciones previas se han analizado el papel que desempeñaron los determinantes medioambientales en la endemia de tracoma que afectó a la España contemporánea ${ }^{5}$, así como los presupuestos conceptuales y metodológicos que guiaron la lucha social contra el tracoma en la España de las décadas de 1920 y 1930, con una atención particular a su mayor incidencia en el mundo rural y al análisis de los factores asociados a dicho medio que determinaban su prevalencia ${ }^{6}$.

El presente trabajo analiza, en primer lugar, y en el contexto de la endemia contemporánea de tracoma que afectó a diversas regiones españolas durante la primera mitad del siglo XX, los factores que explican la prevalencia que adquirió el tracoma infantil y el papel que jugaron los determinantes epidemiológicos de una patología que aparece muy ligada a factores de naturaleza socioeconómica y medioambiental.

3 ArJona y AlONSO (1941), p. 13.

4 ARJONA y AlONSO (1941), p. 14. Tras la guerra civil, y particularmente durante los primeros años de posguerra, el deterioro de las condiciones de vida y los movimientos de población facilitaron la recrudescencia de la endemia de tracoma. En la década de 1960 la enfermedad continuaba siendo un problema de salud pública y mantenía su condición de endémica en doce provincias españolas, aunque el número de casos se había reducido considerablemente. (GIMENO DE SANDE, A. (1964-1965), Memoria sobre la campaña nacional contra el tracoma, Madrid, Ministerio de Gobernación, Dirección General de Sanidad, pp. 109-110).

5 Galiana-SÁnchez, M. ${ }^{a} E$., Cremades Monerris, A. y Bernabeu-Mestre, J. (2007), Environment and health in a proverty-related disease: epidemiology of trachoma in contemporary Spain, 1900-1965. En: Environment Health and History. Conference of the European Association for the History of Medicine and health, London, 12-15th September 2007; BERNABEUMestre, J., Galiana-SÁnchez, M. ${ }^{a}$ E. y Cremades Monerris, A. (2011), Environment and health in a poverty-related disease: epidemiology of trachoma in contemporary Spain, 19001965, História, Ciencias, Saúde - Manguinhos (en proceso de revisión).

6 Galiana-SÁnchez, M. ${ }^{a}$ E., Cremades Monerris, A. y Bernabeu-Mestre, J. (2010), Sanitary campaigns against trachoma in Spain. En: A. ANDRESEN, J.L. BARONA Y S. CHERrY (eds), Making a New Countryside. Health Policies and Practices in European History ca. 1860-1950, Frankfurt am Main, Peter Lang, pp. 101-111. 
En segundo lugar, se abordan las estrategias de intervención que buscaban erradicar la enfermedad tracomatosa desde el modelo de asistencia preventiva que encerraba el concepto de salud comunitaria que se perfiló en el período de entreguerras. Se dedica una atención muy especial a las actividades sobre el medio familiar, escolar y comunitario, y el papel que se otorgaba a los dispensarios de higiene y en particular a las enfermeras de salud pública, en su condición de mediadoras entre el medio sanitario y la población?

La condición de enfermedad crónica y contagiosa que caracteriza al tracoma y el hecho de aparecer sus principales secuelas en la edad adulta ${ }^{8}$, explica que se le haya prestado menos atención a su dimensión de dolencia infantil. Sin embargo, la historia natural de la enfermedad tracomatosa otorga a las primoinfecciones que aparecen durante la edad infantil una gran trascendencia desde el punto de vista social, epidemiológico y terapéutico ${ }^{9}$ : «De esto podemos deducir que la edad en la que el tracoma se adquiere, es en la infancia, especialmente en la primera y en la edad escolar, siendo muy escasas las primoinfecciones en la edad adulta». Como tendremos ocasión de comprobar, el diagnóstico precoz de la infección tracomatosa en la infancia, no sólo evitaba las complicaciones y discapacidades que aparecían en las edades adultas, sino que permitía una acción terapéutica más eficaz.

Como principales fuentes de estudio hemos utilizado los folletos de divulgación, monografías, artículos y comunicaciones a congresos, que fueron publicados por la Dirección General de Sanidad o por el personal del Cuerpo Nacional de Sanidad que tenía responsabilidades en la lucha antitracomatosa, además de los trabajos que dedicaron al tema diversos oftalmólogos y en particular los que lo abordaron desde su condición de problema de salud pública.

7 Véase a este respecto, referido al ámbito de la alimentación y la nutrición, el trabajo: GALIANA-SÁnCheZ, M. ${ }^{\circledR} E$. y Bernabeu-Mestre, J. (2011), Alimentación, enfermería y cultura: el antecedente histórico de las visitadoras puericultoras. En: Alimentación, cultura y salud. Encuentros interdisciplinares, Tarragona, Publicacions de la URV (Col·lecció d'Antropologia Mèdica), en prensa.

8 Socias, A. y Poveda, E. (1935), De los factores endémicos del tracoma. Su estudio en el término municipal de Murcia, Rev. San. Hig. Pub., 11 (6), pp. 1-28, pp. 13-17.

9 Socias, A. Y Delgado, J. (1935), Aportaciones a la epidemiología del tracoma, Rev. San. Hig. Pub., 10 (6), pp. 1-11, p. 4. 
2. UNA ENFERMEDAD DE LA MISERIA: LOS DETERMINANTES EPIDEMIOLÓFICOS DEL TRACOMA

Con la expresión «enfermedad de la miseria» ${ }^{10}$, se pretendía resumir el conjunto de determinantes que estaban detrás del problema del tracoma y que se agrupaban, en definitiva, en torno a un único elemento: las condiciones de vida paupérrimas que sufría una parte importante de la población española de los primeros decenios del siglo XX, en particular la rural ${ }^{11}$.

En los trabajos consultados ${ }^{12}$, se establecían tres grandes grupos de determinantes en el problema epidemiológico del tracoma: los relacionados con la biología humana, los relacionados con las condiciones medioambientales del entorno, y un tercer grupo de factores de naturaleza socioeconómica, demográfica e higiénica ${ }^{13}$.

En el primer grupo de factores se incluía a un conjunto de circunstancias que influían en el estado general del individuo, desde las infecciones a los problemas de alimentación ${ }^{14}$, y que, en opinión de los autores estudiados, podían favorecer el contagio tracomatoso. En el segundo grupo de determinantes, se situaban los factores relacionados con el entorno medioambiental como el clima, la constitución geológica del suelo y la orografía y los elemen-

10 RABAdÁn FernÁndeZ, P. (1936), Factores epidemiológicos en la zona tracomatosa de la provincia de Alicante, Rev. San. Hig. Pub., 1936, pp. 416-422, p. 416. Al valorar la evolución de la endemia tracomatosa en España (DomínguEZ, A. (1955), Lo que todo médico debe saber respecto al tracoma, Murcia, Instituto Provincial de Sanidad, p. 22; MARín AMAT, M. (1958), La lucha antitracomatosa en España y sus satisfactorios resultados, Archivos de la Sociedad Oftalmológica Hispano-Americana, 18 (9), pp. 992-996, pp. 992-993), se destacaba que el control y la erradicación del tracoma había sido posible en la medida en que se consiguieron mejorar las condiciones higiénico-sanitarias y socioeconómicas: «agua abundante y buena, alejamiento higiénico de excretas e inmundicias, alimentación adecuada y suficiente, higiene de las viviendas y de los servicios públicos, instrucción sanitaria por todos los medios conocidos...».

11 Galiana-SÁnchez, M. ${ }^{2} E$. y Bernabeu-Mestre, J. (2006), El problema sanitario de España: saneamiento y medio rural en los primeros decenios del siglo XX, Asclepio, 58 (2), pp. 139-164; RodríGuez OCAÑA, E. (2010), Salud Pública y política agraria liberal en España. La Inspección de Sanidad del Campo (1910-1918), Asclepio, 52 (2), pp. 327-352.

12 Socias y Poveda (1935), p. 27; Socias y Delgado (1935); Socias, A. y Delgado, J. (1939), Estudio de los factores epidémicos en la endemia tracomatosa, Rev. San. Hig. Pub., 13 (11-12), pp. 3-31.

13 ARJONA y Alonso (1941), pp. 71-77.

14 La herencia también se consideraba un elemento a tener en cuenta, llegándose a afirmar que «el tracoma no es hereditario, pero sí las taras que lo favorecen» (ARJONA Y ALONSO (1941), p. 77). 
tos vinculados con el ciclo del agua. En la última categoría de determinantes estarían incluidas las condiciones de la vivienda, los hábitos de vida de la población, la actividad laboral y productiva y los movimientos de población, además de las situaciones de hacinamiento y agregación. Como se indicaba en un trabajo publicado en la Revista de Sanidad e Higiene Pública ${ }^{15}$ : «El tracoma es una enfermedad que de un modo muy especial ataca los barrios pobres, cuya pobreza llega a miseria y cuyas condiciones de vida son el hacinamiento y el hambre».

En el caso de la población infantil las primoinfecciones tracomatosas estaban determinadas por muchos de los factores que acabamos de enumerar, pero eran el medio familiar y escolar los escenarios en los que se desarrollaban y donde se centraron las principales actividades de carácter preventivo y terapéutico ${ }^{16}$.

En el medio familiar ${ }^{17}$, la madre tracomatosa estaba considerada la principal transmisora de la enfermedad, a través del contagio directo. El de carácter indirecto, tenía lugar a través de fómites o vectores y en el contexto del ambiente de miseria que solía rodear a los niños afectados de tracoma. El bajo nivel social de las colectividades más afectadas por la endemia tracomatosa, se sumaba a la insalubridad, asociada fundamentalmente a las deficiencias del ciclo del agua, y a la falta de higiene. La precariedad con la que se veían obligadas a vivir la mayoría de las familias infectadas, se traducía en una ausencia de útiles de aseo individual (toallas, etc.) y la frecuente contaminación de los existentes, favoreciendo el contagio del individuo enfermo al sano. La ignorancia y la negligencia ${ }^{18}$, estaban detrás de prácticas de riesgo como las que llevaban a cabo las madres cuando «limpiaban la secreción purulenta de los ojos de sus hijos con su pañuelo o el vuelco del delantal», y con ese mismo pañuelo o delantal repetían la misma operación con cualquiera de sus otros hijos o con sus mismos ojos.

Las moscas jugaban un papel fundamental en la transmisión de la enfermedad $^{19}$. Su presencia era habitual en aquellos contextos de miseria, donde además se añadía el factor agravante de la convivencia con los animales do-

15 Socias y Delgado (1939), p. 9.

16 Selfa Martínez, E. (1940), Consideraciones sobre la lucha contra las causas de ceguera, Rev. San. Hig. Pub., 1940, pp. 163-177.

17 Socias y Delgado (1939), p. 26; García SANTA-CruZ (1944), Importancia del tracoma en la infancia, Madrid, Delegación Nacional de Auxilio Social, pp. 3-4.

18 Selfa MartíneZ (1940), p. 172.

19 Selfa Martínez (1940), p. 172. 
mésticos y con los estercoleros que generaban sus excreciones ${ }^{20}$ : «El mecanismo de propagación es sencillo. Las moscas que se posan en las comisuras lacrimosas y barnizadas de secreción infectante de los enfermos, recogen en sus patas y trompa buen número de gérmenes, que irán a depositar en los párpados de un sujeto sano o en objetos de uso personal».

Junto al medio familiar, a medida que avanzaba la edad de los niños, adquiría relevancia el medio escolar, aquello que en la literatura de la época se conocía como el factor agregación ${ }^{21}$. La principal fuente de infección la constituían los enfermos y en particular aquellos que mostraban cuadros asintomáticos o lo que se conocía como tracoma oculto, y que se daba sobre todo en la edad escolar ${ }^{22}$. De ahí la importancia que tenían las condiciones de hacinamiento y promiscuidad que mostraban muchas escuelas, y en particular las ubicadas en el ámbito rural ${ }^{23}$.

3. TERAPÉUTICA, PREVENCIÓN Y EDUCACIÓN EN LA LUCHA CONTRA EL TRACOMA INFANTIL: EL MEDIO FAMILIAR, ESCOLAR Y SOCIAL

A partir de las reformas introducidas en la lucha antitracomatosa por la Segunda República en $1933^{24}$, la profilaxis del tracoma infantil se basaba en una actuación coordinada entre el dispensario de higiene, elemento básico en las actividades de salud comunitaria que se empezaron a desarrollar en España en las décadas de 1920 y $1930^{25}$, y la intervención en el medio escolar, mediante el establecimiento de grupos escolares o clases especiales donde eran agrupados los niños tracomatosos ${ }^{26}$. Se trataba de una estrategia de intervención similar a la desarrollada en otros contextos de la Europa mediterránea

20 SOCIAS y POVEDA (1935), p. 24.

21 García SANTA-CruZ (1944), p. 3.

22 DOMÍNGUEZ (1955), p. 15.

23 GALiAnA-SÁNCHEZ Y BeRnABeu-Mestre (2006), pp. 51-53.

24 BARONA VILAR, C. (2006), Las políticas de la salud. La sanidad valenciana entre 1855 y 1936, Valencia, PUV, pp. 173-178.

25 Barona Vilar, J.LL. y Bernabeu-Mestre, J. (2008), La salud y el Estado. El movimiento sanitario internacional y la administración española (1951-1945), Valencia, PUV, pp. 232-233; BARONA VILAR, J.LL. (2009), La sanitat rural, una qüestió d'estat. En: X. GuILLEMLlobat y G. GARCía FrasQuet (eds), Salut, alimentació i cultura popular al País Valencià, Gandia, Ceic, Alfons el Vell, 2009, pp. 13-37; GALIANA-SÁnCheZ, CremadeS y BernabeuMestre (2010), pp. 106-109.

26 Selfa MartíneZ (1940), p. 172. 
donde la enfermedad tracomatosa también era endémica ${ }^{27}$, y que debe situarse en el marco de las iniciativas internacionales que se habían venido estableciendo en la luchas y campañas sanitarias ${ }^{28}$.

La creación de clases especiales para niños tracomatosos pero sin aislarlos del medio escolar y social, representaba una medida complementaria a las escuelas-sanatorios o los asilos-hospitales para tracomatosos, ${ }^{29} \mathrm{y}$ una alternativa para las medidas aisladas de segregación que se venían practicando y que tenían consecuencias negativas tanto para los niños tracomatosos como para la colectividad. Como se recogía en un texto de 1923 sobre «El tracoma y la Escuela», presentado en el II Congreso Nacional de Pediatría, cuando se impedía a los niños infectados el acceso a la escuela, además de no garantizar su derecho a la educación ${ }^{30}$, se convertían en «un peligro de contagio» al no existir ningún tipo de control sanitario ${ }^{31}$ :

La simple expulsión hasta su curación, entre otros inconvenientes en los aspectos didáctico y moral, tiene el gravísimo de que este núcleo de tracomatosos queda sin control sanitario eficaz, ya que por regla general no se someten voluntariamente a tratamiento adecuado. Estos niños, faltos de escuela, se dedican entonces a sus juegos favoritos en calles y paseos, con lo que las posibilidades de contagio en la calle aumentan.

Cuando la enfermedad se lo permitía, muchos de aquellos niños sin escolarizar eran contratados como sirvientes, lavanderas, camareros y otros oficios

27 Melis, P. y PozzI, L. (2010), Trachoma, health conditions and social change in Sardinia. En: Andresen, A., BArona Vilar, J.L. y Cherry, S. (eds), Making a New Countryside. Health Policies and Practices in European History ca. 1860-1950, Frankfurt am Main, Peter Lang, pp. 25-48.

28 BOROWY, I. Y GRUNER, W.D. (eds.) (2005), Facing illness in troubled times. Health in Europe in the interwar years, 1918-1939, Frankfurt am Main, Peter Lang; RODRÍGUEZ OCAÑA, E. Y ZYLBERMAN, P. (2008), Improving public health amidst crises. Introduction, Dynamis, 28, pp.19-27.

29 Con estas instituciones se pretendía aislar a los enfermos de los sanos, someterlos a tratamiento, educarlos e instruirlos para saber aplicar las normas higiénicas necesarias para no transmitir la infección, y ofrecerles la formación necesaria para poder desempeñar oficios compatibles con su enfermedad y «que no ofrezca peligro, ni para sus compañeros de trabajo, ni para los demás conciudadanos, y al mismo tiempo les proporcione medios de vida suficientes para si y para los suyos» (Soriano Fischer, E. (1924), El tracoma y la escuela. En: Actas del Congreso Nacional de Pediatría, Madrid, Hospital del Niño Jesús, 1924, IV, p. 171-174, p. 173.

30 SORIANO FISCHER (1924), p. 172.

31 Selfa Martínez (1940), p. 167. 
de fácil aprendizaje, pero que por el contacto constante con los sanos ponía a estos en peligro de contraer la enfermedad ${ }^{32}$ :

¡Que frecuente es en las consultas públicas de ojos encontrar niñeras, planchadoras, etc., que padecen tracoma y que son un constante peligro para los niños que llevan o para las personas cuya ropa cuidan! Es decir, alejamos a estos enfermos de las grandes colectividades, pero para nada nos ocupamos de su aislamiento, ni de su educación ética, que es la que fundamentalmente evitaría estos casos de verdadero delito sanitario.

Las clases especiales para tracomatosos permitían resolver, de hecho, muchos de aquellos problemas. Su creación en las zonas donde la enfermedad era endémica, completaba y hacía más eficaz la labor profiláctica que llevaban a cabo los dispensarios de higiene ${ }^{33}$, y no representaba un sobreesfuerzo presupuestario ni organizativo. Como hemos apuntado con anterioridad, la población infantil era considerada el reservorio de la enfermedad tracomatosa, pero al mismo tiempo presentaba «una cualidad inestimable»: su fácil curabilidad $^{34}$. Se debían elegir las aulas con mejores condiciones de ventilación e iluminación, y además de seleccionar a personal docente no tracomatoso como responsables de las mismas, se les proporcionaba los medios necesarios para proceder a la cura diaria de los niños tracomatosos ${ }^{35}$.

La intervención sobre el medio escolar (véase esquema), era supervisada desde el ámbito sanitario a través de las enfermeras visitadoras. Además de proporcionar continuidad a la inspección médico-escolar, a las intervenciones de los médicos locales y de los especialistas (oftalmólogos), y hacerse cargo de la educación higiénico-sanitaria, su labor permitía iniciar una actuación en el ámbito familiar y comunitario. Para cada alumno tracomatoso, las enfermeras abrían una ficha familiar y llevaban a cabo las visitas domiciliarias que permitían una evaluación de los factores de riesgo y la detección de los otros miembros de la unidad familiar que estaban afectados por la enfermedad, con lo que se podía proceder a su vigilancia desde el dispensario de higiene y someterlos a control y tratamiento, además de reeducarlos en cuestiones de

32 SORIANO FISCHER (1924), pp. 171-172.

33 Barona Vilar (2006), pp. 171-178; Galiana-SÁnchez, Cremades y BernabeuMeSTRE (2010), pp. 106-107.

34 Selfa Martínez (1940), pp. 164-165.

35 Selfa MARTínez (1940), pp. 167-168. Las curas las llevaban a cabo los maestros y maestras, asesorados y supervisados por las enfermeras visitadoras, y para ello contaban con un lavabo, algodón, alcohol y colirios de hermofenil al 1\% y sulfato de zinc al 5\%. 
hábitos y conductas higiénico-sanitarias ${ }^{36}$. Se trataba de controlar aquello que en el lenguaje epidemiológico se conocía como los reservorios familiares ${ }^{37}$. Unos reservorios que, como hemos indicado de forma repetida, aparecían casi siempre ligados a la suciedad que acompañaba la miseria ${ }^{38}$.

\section{ESQUEMA DE INTERVENCIÓN EN EL MEDIO ESCOLAR, FAMILIAR Y SOCIAL}

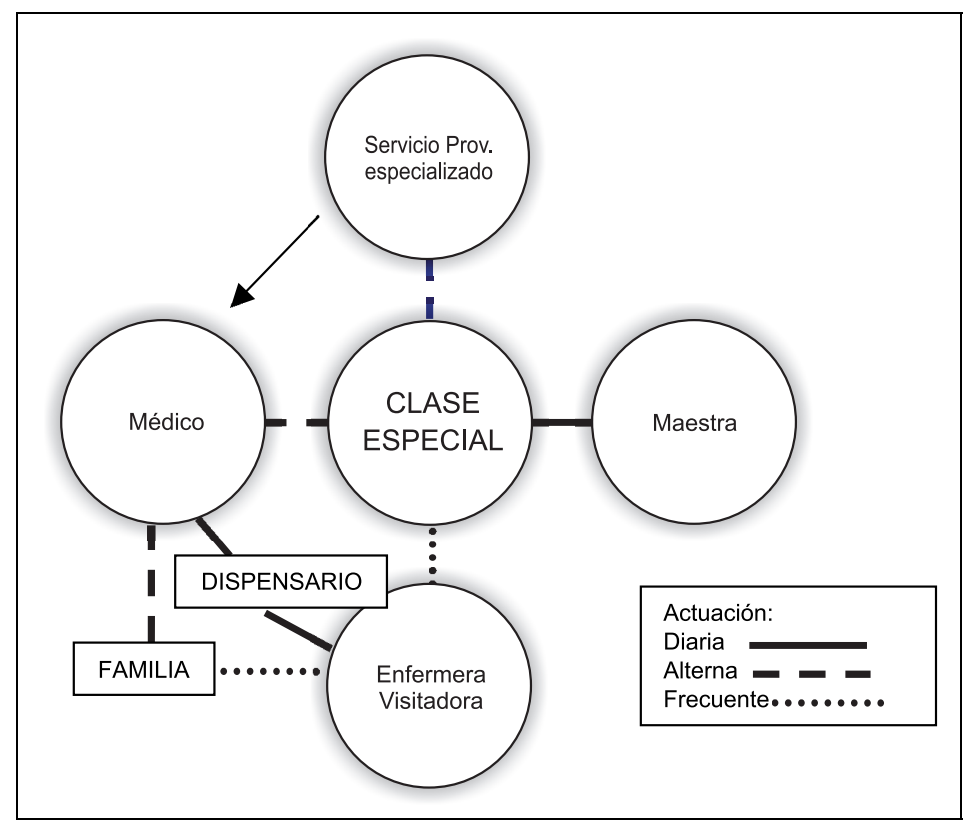

Fuente: Elaboración propia.

\footnotetext{
36 Socias y Delgado (1939), p. 4; Selfa Martínez (1940), pp. 167-168.

37 SOCIAS Y POVEDA (1935), p. 16.

38 Como señalaban Socias Y Delgado (1939, p. 24): «De todo lo antes expuesto claramente se deduce el papel capital del contagio de los hijos por los padres, y repitamos una vez más que es preciso tener siempre en cuenta las circunstancias de lugar y tiempo en que se produce una endemia con sus características para ser comparada con otras. La endemia tracomatosa se extiende lenta pero paulatinamente, siempre y cuando el medio que encuentra reúna las condiciones indispensables a su propagación y una de éstas, como venimos diciendo, es el factor miseria, y en tales circunstancias la difusión endémica de la enfermedad se hace rápidamente en unas generaciones, no sucediendo lo propio cuando el medio ambiental es el de una familia acomodada y limpia, donde se toman los cuidados higiénicos individuales elementales; en tal caso, la enfermedad no se extiende endémicamente en medio de tal población».
} 
La clave consistía en lograr interrumpir la cadena de contagio y evitar que los casos pasaran de la familia a la escuela y viceversa. Como ocurría en la mayoría de las enfermedades denominadas sociales, la labor estrictamente sanitaria era insuficiente y los profesionales de la salud debían llevar a cabo una autentica labor social. Las enfermeras visitadoras, en este sentido, jugaron un papel crucial al actuar como enlaces entre los tres ámbitos implicados: por un lado la población escolar y los maestros, por otro las instituciones sanitarias, médicos y servicios especializados, y por otro las familias y población general ${ }^{39}$.

Desde principios del siglo XX, y sobre todo durante el periodo republicano, la formación y el desempeño laboral de las enfermeras comunitarias en España puso su acento en esta labor social, que se consideró imprescindible desde la perspectiva higienista ${ }^{40}$. Así parece que ocurrió con los dispensarios antitracomatosos ${ }^{41}$. A través de las visitas domiciliarias, se buscaba actuar sobre el principal determinante de la enfermedad: la influencia del medio ambiente y específicamente la importancia de la vivienda. En estas visitas, las enfermeras, cuya formación había sido orientada específicamente en el estudio de la endemia tracomatosa, debían cumplimentar las fichas familiares ${ }^{42}$, que permitían la elaboración del diagnostico del tracoma y en las que se recogía información relevante sobre la situación de salud de la familia. Además, a través de estas visitas, se podían llevar a cabo actuaciones de educación sanitaria sobre aspectos higiénicos y mecanismos para controlar y evitar el contagio de la enfermedad. Finalmente, cabe mencionar la contribución singular de su actuación. Las visitadoras eran consideradas «consejeras y mentoras de la vida cotidiana de las familias» ${ }^{43}$. Su trabajo se caracterizaba por su cercanía con la población a atender, estableciendo importantes vínculos con las personas enfermas y con el conjunto de la comunidad. Esta vertiente de su labor, su papel de mediadoras y su proximidad con las familias ${ }^{44}$, ha permanecido en

39 «La educación sanitaria tenía que crear conciencia de la gravedad de la infección e informar sobre los principales medios de lucha. Los profesionales sanitarios (médicos y enfermeras) tenían que formar a los maestros y estos a sus alumnos con tanto mayor interés en cuanto que en los adultos la curación es ya más difícil. Debían utilizarse todos los medios de propaganda: prensa, carteles, folletos, radio, cine, conferencias, etc.» (DOMÍNGUEZ (1955), 21).

40 GASCón-PÉrez, E, Galiana-SÁnCHEZ, M. ${ }^{a}$ E. y BernabeU-Mestre, J. (2002), La acción social de las visitadoras e instructoras sanitaria, Revista de Trabajo Social y Salud, 43, pp. 149-174.

41 Selfa Martínez (1940), p. 64.

42 SOCIAS Y DELGado (1939), pp. 4-9.

43 Martin Yumar, D. y Huertas MúgicA, E. (1933), La mortalidad infantil en el ambiente rural, Rev. San. Hig. Pub., 1933, pp. 470-485, p. 482.

44 Selfa Martínez (1940), p. 169. 
cierto modo invisible y, sin embargo, parece que resultó relevante en la mejora de la salud comunitaria en general, tal como se refleja en acciones como las que llevaron a cabo en la endemia de tracoma que sufría la población española en las décadas de 1930 y 1940.

El modelo de intervención profiláctica/preventiva que acabamos de describir, tuvo una cierta continuidad durante los primeros años del franquismo, pero el freno que padecieron las políticas de salud pública ${ }^{45}$, o el proceso de desinstitucionalización al que fueron sometidos colectivos como el de la enfermería comunitaria ${ }^{46}$, sumado a la aparición de la antibióticoterapia, hicieron que la lucha contra el tracoma dejase de pivotar sobre la barrera profiláctica y adquiriera un mayor protagonismo la barrera terapéutica. ${ }^{47}$ Como se recogía en la memoria sobre la Campaña Nacional de Lucha contra el Tracoma que se llevó a cabo en los inicios de la década de $1960^{48}$, aunque formalmente se cumplimentaban las fichas familiares del modelo OMS/UNICEF donde se recogían datos sobre situación económica social, condición ambiental e higiénico sanitaria de la vivienda, o los datos clínicos de los miembros de la unidad familiar que estaban afectados, la figura profesional de las visitadoras fue sustituida por «señoritas auxiliares previamente entrenadas» pero sin formación y capacitación sanitaria, y que se limitaban a dar soporte a las tareas administrativas y ayudar en el tratamiento, que consistía en la aplicación intermitente de pomada de aureomicina al $1 \% 49$.

45 Rodríguez Ocaña, E. y Martínez Navarro, F. (2008), Salud Pública en España. De la Edad Media al siglo XXI, Escuela Andaluza de Salud Pública, Consejería de Salud, pp. 85-87.

46 Galiana-Sánchez, M. ${ }^{a}$ E., García-Paramio, P. y Bernabeu-Mestre, J. (2009a), Luchando por la supervivencia: Primera Asamblea Nacional de Enfermeras (Madrid, 1959), Temperamentvm, 8, disponible en http://www.index-f.com/temperamentum/tn8/t6786.php (consultado el 10-11-2011); GAliAnA-SÁncheZ, M. ${ }^{a}$ E., García-PARAmio, P. y Bernabeu-MeStre, J. (2009b), La frustración de una profesión: la enfermería de salud pública en la V Reunión de Sanitarios, 1959, Temperamentvm, 9, disponible en http://www.index-f.com/temperamentum/tn9/ t2408.php (consultado el 10-11-2011); GALIANA-SÁNCHEZ, M. ${ }^{\mathrm{a} E ., ~ B E R N A B E U-M E S T R E, ~ J . ~ Y ~ G A R-~}$ CÍA-PARAMIO, P. (2010), Enfermeras para una nueva patria. Género e ideología en la enfermería de Falange. En: C. GONZÁlEZ y F. MARTíNEZ (eds.), La transformación de la enfermería. Nuevas miradas para la historia, Granada, Comares, pp. 203-216.

47 Es la misma situación que se reprodujo en otras campañas sanitarias, y en concreto en el caso de la mortalidad infantil (Bernabeu-Mestre, J., CABAllero, P., GalianA-SÁnchez, M. ${ }^{a}$ E. Y NolASCO, A. (2006), Niveles de vida y salud en la España del primer franquismo: las desigualdades en la mortalidad infantil, Revista de Demografia Histórica, 24 (1/2), pp. 173-193).

48 GIMENO DE SANDE (1964), p. 12-14.

49 Gimeno DE SANDE (1964), p. 67-68. 


\section{CONCLUSIONES}

El trabajo ha permitido ampliar el conocimiento historiográfico sobre el desarrollo que llegó a alcanzar en la España de la década de 1930, el modelo de asistencia preventiva que encerraba el concepto de salud comunitaria que se perfiló en el período de entreguerras. La detección precoz de casos de tracoma infantil en el ámbito escolar era considerada una estrategia eficaz para poder actuar sobre el ámbito familiar y comunitario. El control sanitario de la enfermedad, supervisado desde los dispensarios y centros de higiene, tenía que ser ejecutado a través de la profilaxis y las actividades preventivas, educativas, terapéuticas y de inspección. En todas aquellas intervenciones, resultaba fundamental el trabajo que debían llevar a cabo las visitadoras sanitarias, a través de la búsqueda activa y la detección precoz de casos y la modificación de algunos de los factores de riesgo que aparecían ligados a la insalubridad de las viviendas y a la falta de hábitos higiénicos de la población.

La experiencia histórica española de lucha contra el tracoma infantil que acabamos de resumir, permite reafirmar la validez de aquellas estrategias que sostienen que para poder acabar con el problema de salud que representa la enfermedad tracomatosa en las zonas menos desarrolladas y pobres del planeta, no basta con actuar sobre los factores más inmediatos, a través de los recursos terapéuticos. Por el contrario, es necesario mejorar las condiciones higiénicas y los factores medioambientales que explican su prevalencia, a través de una intervención horizontal sobre el ámbito familiar y comunitario ${ }^{50}$.

Recibido: 3 de marzo de 2011.

Aceptado: 12 de julio de 2011.

50 MARIOTTI Y PRÜSS (2000); Wright, TURNER Y TAYLOR (2008), p. 1945. 